\title{
Prototipo de una aplicación móvil accesible para apoyar a la localización de adultos mayores mediante un código compartido entre usuarios
}

\author{
Etelvina Archundia, Carmen Cerón, Beatriz Beltrán, Patricia Cervantes, \\ Fernando Rodríguez \\ Benemérita Universidad Autónoma de Puebla, \\ Facultad de Ciencias de la Computación, México \\ \{etelvina, mceron, bbeltran\} @cs.buap.mx, \\ \{cervantes.patty, ferrgzt\} egmail.com
}

\begin{abstract}
Resumen. El propósito de este artículo es presentar el diseño e implementación de una aplicación móvil para distintos dispositivos con sistema Android que permita apoyar la localización de adultos mayores mediante el uso de un código, que hace referencia a la longitud y latitud del usuario, estos códigos estarán almacenados en una base de datos que se consultaran mediante un Web Service. La aplicación puede compartir el código entre distintos usuarios y dispositivos para que puedan ver la localización correspondiente del adulto mayor brindando mayor protección y seguridad en el uso de la información y de la tecnología. El desarrollo de la aplicación móvil se basó en la metodología Extreme Programación, el Diseño de Centrado en el Usuario, y las tecnologías integradas Web Service, PHP, MYSQL, Java y Apache. Finalmente se presenta los resultados obtenidos de las pruebas de usabilidad con una muestra de usuarios adultos mayores bajo distintos escenarios de prueba.
\end{abstract}

Palabras clave: Servicios web, apps, usabilidad, tercera edad.

\section{Prototype of Application Mobile Accessible for Bringing Support to the Localization of Elderly People through a Code that is Shared between the Users}

\begin{abstract}
The purpose of this article is to introduce the design and implementation of a mobile app in gadgets that work using Android system to support the location of elderly people who have any disability using a code and share it with other apps, this code will show the user length and altitude and these data will be stored in a database that will be consulted in a Web Service. The app will be able to share the code between many users and apps and therefore the user will be able to watch the location of an elderly person providing more safety and the use of technology. The developing of this mobile app was based in
\end{abstract}


Extreme Programming methodology, Design Focused in the User and Integrated Technologies in a Web Service, PHP, MYSQL, Java and Apache. Finally, it is introduced the results that were obtained by usability testing with a sample of elderly users under different test scenarios.

Keywords. Web service, apps, usability, elderly people.

\section{Introducción}

Las aplicaciones tecnológicas para los adultos mayores en ocasiones representan dificultades para poder utilizarlas de forma sencilla por la falta de usabilidad de las interfaces y la carga cognitiva que requieren, lo cual ha provocado una creciente brecha digital generacional entre la tecnología y los adultos mayores, lo cual ha identificado diferentes grupos de generaciones en función de su capacidad de comunicación a través de las herramientas tecnológicas [1].

Las tecnologías deben brindar mejores alternativas de comunicación y apoyar las actividades cotidianas de los adultos mayores, para aportar una mayor calidad de vida en esta población vulnerable, la cual demanda apoyo, protección y seguridad. Es por eso que dentro de los servicios a los grupos vulnerables por parte de la Institución se lleva a cabo un proyecto de atender a personas discapacitadas, adultos mayores y población indígena. Una de las problemáticas que se han identificado en adultos mayores es la discapacidad auditiva o visual, por lo cual es necesario proporcionar herramientas o artefactos tecnológicos para apoyarlos en su entorno de trabajo, familiar y social. A finales del 2013 el organismo internacional de las Naciones Unidas ha reportado que para el año 2050 estiman más de 2000 millones de adultos mayores usarán Internet, siendo un aumento del 300\% [2]. Esto conlleva que el crecimiento de esta población hará uso de distintas aplicaciones y dispositivos tecnológicos con mayor frecuencia en las actividades cotidianas y que requieren ser adaptadas a sus necesidades y condiciones personales.

En la actualidad por medio de las redes sociales y de distintas aplicaciones móviles se puede compartir información, siendo uno de los principales problemas actuales la seguridad de la información. En el caso de los adultos mayores el acceso a la tecnología no es fácil por la complejidad que muestran las interfaces y en especial las aplicaciones de localización, siendo este grupo vulnerable a extraviarse y requerir ayuda.

El presente trabajo tiene como propósito el diseño y desarrollo de una Aplicación Móvil para dispositivos con sistema Android con la finalidad de compartir la ubicación de manera rápida y sencilla mediante un código y el uso de interfaces accesibles para el Adulto Mayor, para estar disponible en el Portal de Servicios a Grupos Vulnerables de la Institución como parte de un proyecto comunitario y social.

La aplicación permitirá ubicar al usuario de manera exacta y en caso de moverse, el usuario podrá arrastrar el marcador para hacer coincidir con la ubicación, generar un código único, actualizado y almacenarlo en la base de datos. El Web Service permitirá la obtención de la información y mostrar la ubicación real del usuario adulto con los usuarios que se compartió la información, logrando el uso del dispositivo móvil de forma más accesible, segura mediante interfaces intuitivas y sencillas. 
Prototipo de una aplicación móvil accesible para apoyar a la localización de adultos mayores ...

El artículo se organiza de la siguiente manera: En la sección 2 presenta la fundamentación teórica y el estado del arte de este trabajo. En la sección 3 se define el análisis y diseño de la aplicación móvil. En la sección 4 Se presenta la propuesta de la aplicación móvil, la implementación y pruebas de usabilidad. Finalmente, las conclusiones y el trabajo futuro se presentan en la sección 5.

\section{Marco teórico}

En esta sección revisaremos los tópicos de discapacidad y la tecnología, aplicaciones móviles y el diseño centrado en el usuario, los cuales son relevantes en nuestra propuesta de este trabajo.

\subsection{Discapacidad y tecnologías}

En general la tecnología ha tenido que proponer adaptaciones para poder ser incluyente y accesible a personas con distintas discapacidades. Por otra parte, los adultos mayores requieren ser alfabetizados en el uso de las Tecnologías de Información y Comunicación. En ese sentido ha surgido la Tecnología de Apoyo conocida también como "tecnología de adaptación o rehabilitación". Según Alcantud la define como "Todos aquellos aparatos, utensilios, herramientas, programas de ordenador o servicios de apoyo que tienen como objetivo incrementar las capacidades de las personas que, por cualquier circunstancia, no alcanzan los niveles medios de ejecución que por su edad y sexo le corresponderían en relación con la población normal" [3]. De acuerdo al Artículo 2 de la Convención de Naciones Unidas sobre los Derechos de las Personas con Discapacidad, aprobada en 2006 [4], existe la necesidad que se diseñen aplicaciones bajo un diseño universal para todos pero que no excluye las ayudas técnicas para grupo particulares de personas con discapacidad, cuando se necesiten que se adapten a las capacidades específicas y que con respecto a la accesibilidad se enfatice tanto para contenidos y servicios como para herramientas y dispositivos.

\subsection{Aplicaciones móviles accesibles}

Según Gil [5], las Aplicaciones Móviles o conocidas como Apps, han establecido principios básicos para el diseño de Apps Accesibles de acuerdo con la norma "UNE 139802:2009 - Requisitos de accesibilidad del software. (ISO 9241-171:2008)", los cuales han sido adaptados a las necesidades de los dispositivos móviles.

Las aplicaciones móviles accesibles han sido definidas como "una aplicación es accesible cuando cualquier usuario, independientemente de su diversidad funcional, puede utilizarla en su dispositivo móvil satisfactoriamente con su sistema de acceso habitual" [5].

Una aplicación móvil se identifica en la actualidad porque puede funcionar en distintos dispositivos móviles, y existen tres tipos [6]: 
- Aplicaciones nativas, son aquellas que se desarrollan bajo un lenguaje y un entorno de desarrollo en específico para cada sistema operativo.

- Aplicaciones web, estas son desarrolladas usando leguajes para el desarrollo web como lo son HTML, CSS y JavaScript.

- Aplicaciones Hibridas, como su nombre lo indica tienen un poco de cada tipo de las aplicaciones anteriores.

Las aplicaciones móviles las podemos encontrar en las diferentes tiendas como: AppStore, GooglePlay y Windows Phone Store, quienes ofrecen servicios de compras en línea, educación, finanzas, navegación. Las aplicaciones de geolocalización ofrecen servicios para facilitar las tareas como: mostrar el tráfico, optimización de rutas, lugares de interés social, recordatorios al pasar cierta zona, ver los lugares más visitados, buscar direcciones y otros. Las aplicaciones más usadas en este contexto son:

Google Maps es una aplicación móvil de mapeo desarrollado por Google para los sistemas operativos Android e iOS que utiliza la API de Google Maps para su información. Los mapas e información no están incluidos en los mapas de Google instaladas para los dispositivos, se requiere una conexión a Internet para hacer uso de ellos y permite al usuario descargar la ruta de un punto de referencia a otro, con un área de 10 millas cuadradas $(26 \mathrm{~km} 2)$ alrededor de cualquier punto [7].

Waze es la aplicación de tráfico y navegación basada en la comunidad más grande del mundo, dando información de tráfico y ruta en tiempo real por sus usuarios [7].

Foursquare consiste en una red social en la que los usuarios realizan 'check-ins' en los lugares que visitan mientras comparten recomendaciones y opiniones acerca de estos con sus contactos. Para poder hacerlo de manera efectiva hace uso del GPS del dispositivo para realizar check-ins de la forma más sencilla posible [7].

Una aplicación llamada Life 360, es un localizador familiar en dos versiones: básica (gratuita) y Premium (con costo), siendo esta la cual integra mejores servicios de localización, pero el costo es elevado [8].

\subsection{Sistemas de posicionamiento}

Los sistemas de posicionamiento se refieren al método por el cual se puede determinar la posición de un dispositivo, y utilizando servicios adicionales se puede responder preguntas como ¿Dónde estoy?, ¿Qué hay cerca?, ¿Cómo llego allá?, entre otras.

La ubicación puede ser expresada como una descripción textual o en términos espaciales [9]. Una ubicación expresada como un texto es por lo general la dirección de una calle, la ciudad, comuna, código postal, etc. Una ubicación espacial puede ser expresada en términos de coordenadas geográficas usando latitud-longitud-altitud (esta última opcional). La latitud se expresa en grados de 0-90 al norte o sur del ecuador, la longitud en grados de 0-180 al este u oeste del meridiano de Greenwich, y la altitud en metros sobre el nivel del mar. Existen varios métodos [9] para determinar la ubicación de un dispositivo, los cuales varían en las tecnologías empleadas y en la precisión con que entregan el resultado. Siendo las más utilizadas las redes telefónicas, celulares, de área local, Wi-Fi, Bluetooth y GPS entre otros. 
Con respecto al método Cell-ID, es considerado de bajo costo que utiliza la red GSM para determinar la ubicación de un teléfono celular. El identificador de la antena o célula (Cell ID) a la que está conectado el dispositivo es usado para aproximar la ubicación del usuario. La precisión de este método depende del tamaño de la célula: unos 500 metros de exactitud dentro de una ciudad y unos 10 kilómetros en zonas rurales.

Por otra parte, el método Bluetooth, es una red de corto alcance, este provee un posicionamiento de buena precisión, con máximo de error de unos 10 metros, además de poder entregar posicionamiento vertical. Es muy adecuado para obtener la ubicación dentro de edificios o zonas urbanas pequeñas.

\subsection{Diseño centrado en el usuario}

El diseño centrado en el usuario (DCU) es aquel que, desde los inicios del desarrollo del producto, hace participar al usuario y lo involucra durante todo el proceso [10]. La Usability Professionals'Association (UPA) lo define como un enfoque de diseño cuyo proceso está dirigido por información sobre las personas que van a hacer uso del producto, siendo una guía para facilitar el logro de una mayor calidad en el uso de sistemas interactivos que consta de cuatro fases, como se muestra en la Figura 1.

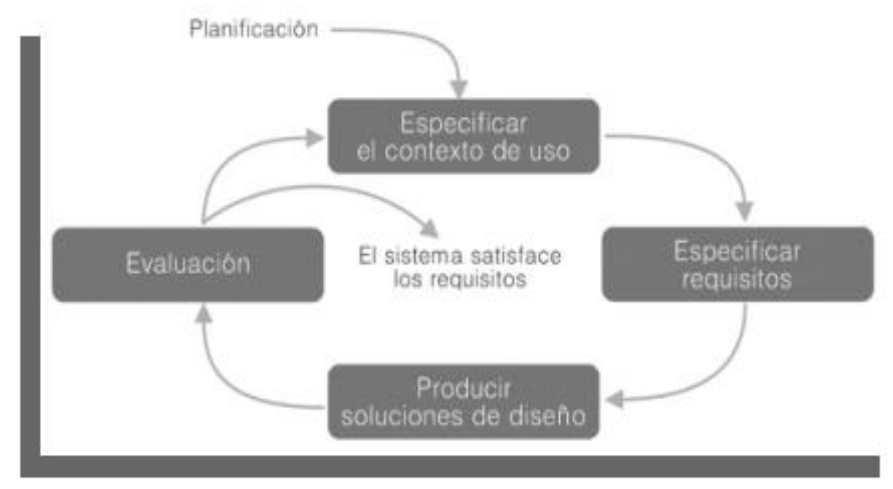

Fig. 1. Proceso del diseño centrado en el usuario.

\section{Análisis y diseño de la aplicación móvil de localización "TENAMIKI"}

En esta sección se presenta el análisis, diseño, la arquitectura de la aplicación y el diseño de la base de datos, bajo el enfoque de la metodología ágil de la Programación Extrema y el diseño centrado en el usuario. 


\subsection{Metodología ágil de la programación extrema}

Para el análisis y el diseño de la aplicación se utilizó la programación Extrema y sus fases [11], ya que permite de forma más sencilla el desarrollo de aplicaciones móviles con la fase de exploración, se determinaron los requerimientos por medio de las Historias de usuarios del grupo focal: "Adultos mayores discapacitados" y se integraron los casos de uso en UML para la aplicación [11].

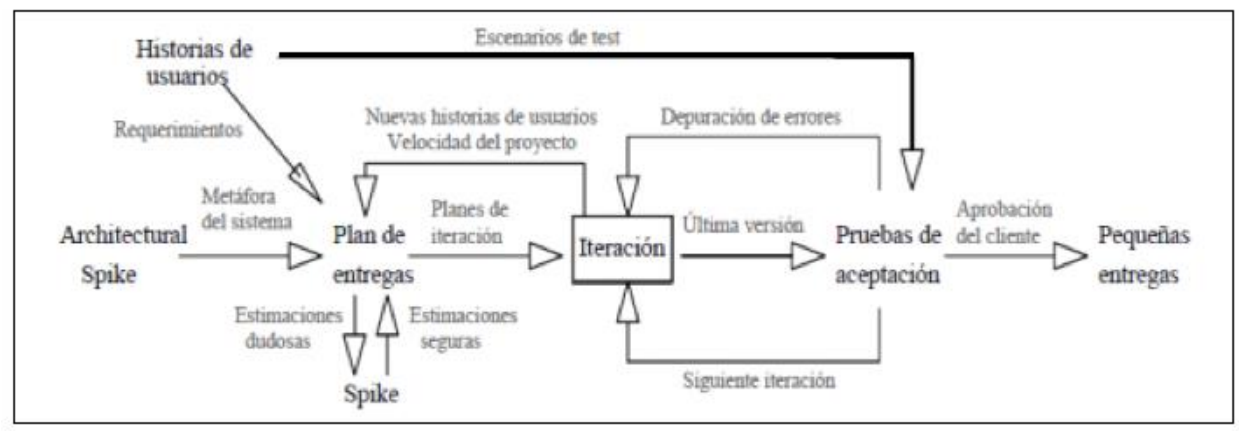

Fig. 2. Ciclo de vida de un proyecto con XP.

En la Figura 3, se muestra al usuario "Adulto Mayor" con las actividades a desempeñar y la interacción que tiene con la base de datos del dispositivo y el Web Service.

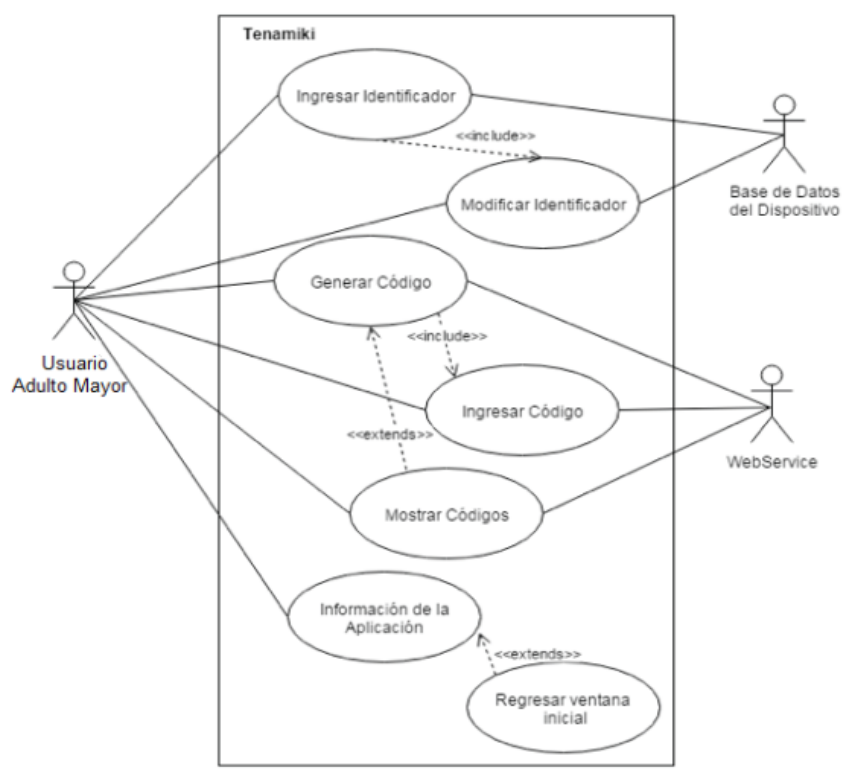

Fig. 3. Caso de uso del sistema. 


\subsection{Arquitectura de la aplicación}

La arquitectura de la aplicación consta de dos partes: la primera parte es la aplicación para dispositivos Android, la cual estará conectada al Web Service [12], que a su vez se comunica con la base de datos que almacena e intercambiar información de la aplicación. Por otra parte, se consta de un módulo Web agregado al servidor web con Apache para el portal de aplicaciones de apoyo a Adultos Mayores Accesibles (AAMA) de la institución, como se muestra en la Figura 4.

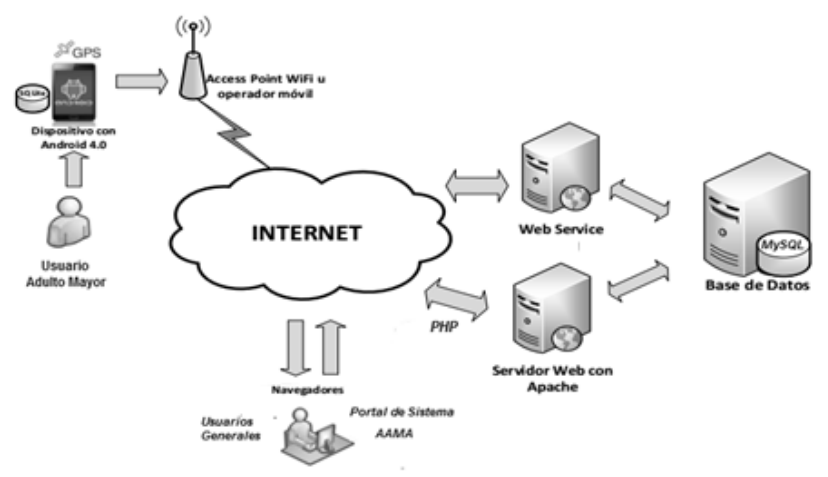

Fig. 4. Esquema de la arquitectura de la Aplicación TENAMIKI.

\subsection{Diseño centrado en el usuario de la aplicación}

El Diseño centrado en el usuario se adoptó a las características propias del usuario de Adulto Mayor y teniendo en cuenta que pueden tener alguna discapacidad auditiva. Así como al desarrollo de aplicaciones móviles que requieren ser accesibles para todos. Para lo cual se realizó una serie de entrevistas y encuestas con el grupo focal, finalmente se plantearán los prototipos de la aplicación, los cuales se realizaron con el Software Balsamic.

Los resultados obtenidos fue que la mayoría de los adultos ya poseen un teléfono inteligente, siendo el sistema operativo que más predomina el Android y de las herramientas que han escuchado y muy poco utilizado es Google Maps y Waze, pero aseguran que requieren no solo compartir su localización, sino tener certeza de que hayan recibido la localización sus familiares o conocidos y saber cuántos usuarios han consultado la ubicación que han compartido y poder llevar un registro incluso de las personas contactadas por este medio y los datos de la localización para dar un servicio de información detallado desde el portal y al usuario de la aplicación. Por lo cual se planteó las características principales con las que debe contar la aplicación:

Interface clara e intuitiva: Puesto que lo han de utilizar personas con muy pocas habilidades tecnológicas, se requiere de una interface gráfica muy clara e intuitiva, con pocos botones y que los que haya sean grandes.

Pocas opciones hasta llegar al resultado: Se quiere que la aplicación sea ágil, así que se necesita pocas pantallas y decisiones, para llegar al resultado esperado. 
Práctica: La aplicación debe resultar lo más práctica posible para que el usuario la encuentre de gran utilidad.

\section{Desarrollo y pruebas del prototipo de la aplicación móvil}

En esta sección se muestran los resultados del prototipo de la implementación de la aplicación móvil, así como las pruebas de funcionalidad junto con las pruebas de usabilidad obtenidas del grupo focal de usuarios. Las tecnologías utilizadas PHP, MySQL, Webservices, Java, Android Studio 15, Servidor Web: Apache 2.4.10, Emulador Android: Genymotio 2.5.3. Las interfaces finales para la aplicación móvil TENAMIKI (significa en náhuatl "Encontrase") como se muestran en las Figura 5.
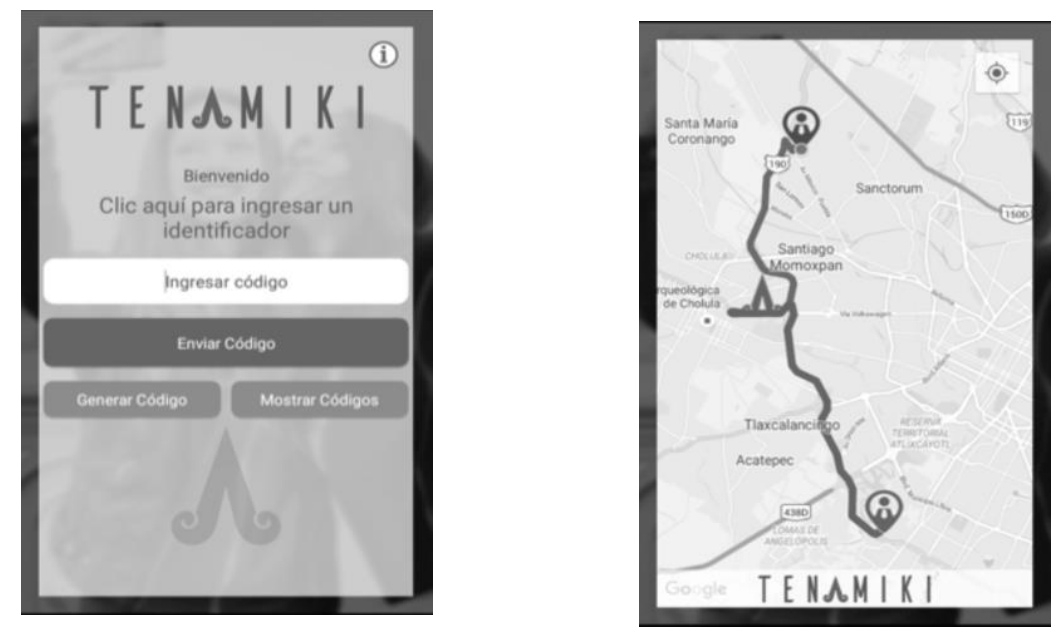

Fig. 5. Interfaces del sistema móvil de ingreso y de acceso al usuario.

\subsection{Prueba piloto y de usabilidad}

La aplicación móvil se le aplicó una prueba piloto un grupo de 15 usuarios adultos mayores, de los cuales el $80 \%$ tiene una disminución auditiva sin llegar a una sordera total. Para la evaluación de la aplicación, se realizaron pruebas de funcionalidad y usabilidad. Con respecto a las pruebas de Usabilidad presentaron tres posibles escenarios posibles para los usuarios finales, a continuación, se describen:

Situación 1: Al usuario se le entregó la aplicación con la WiFi, GPS y 3G apagados, además se les dio una breve explicación del uso de la aplicación.

Situación 2: Al usuario se le entregó la aplicación con la WiFi, GPS y 3G prendidos y se le explico el uso de la aplicación.

Situación 3: Al usuario se le entregó la aplicación con la WiFi, GPS y 3G apagados y no se explicó el uso de la aplicación

Para cada una de las situaciones los usuarios deberán cumplir ciertas tareas para así comprobar el funcionamiento de la aplicación. 
Tarea 1: Ingresar un código generado.

Tarea 2: Encontrar donde se muestran los códigos que se han generado.

Tarea 3: Generar un código en una localización diferente a la que se encuentra actualmente.

Tarea 4: Cambiar el identificador.

Tarea 5: Compartir el código.

Después de realizar las pruebas a 15 usuarios 5 por cada situación, se muestran los resultados obtenidos en la Tabla 1 , donde se observa el porcentaje de usuarios que pudieron completar la tarea.

Tabla 1. Resultados prueba de usabilidad usando escenarios.

\begin{tabular}{cccc}
\hline \multicolumn{3}{c}{ Escenarios de Test } \\
\hline Tareas & Situación 1 & Situación 2 & Situación 3 \\
\hline Tarea no.1 & $90 \%$ & $95 \%$ & $60 \%$ \\
Tarea no.2 & $100 \%$ & $100 \%$ & $100 \%$ \\
Tarea no.3 & $100 \%$ & $100 \%$ & $100 \%$ \\
Tarea no.4 & $100 \%$ & $100 \%$ & $100 \%$ \\
Tarea no.5 & $80 \%$ & $100 \%$ & $80 \%$ \\
Promedio & $94 \%$ & $99 \%$ & $88 \%$ \\
\hline
\end{tabular}

Lo cual refleja que los usuarios con una breve explicación de la Situación 1 su desempeño fue del $94 \%$ del cumplimiento de las tareas mientras que los usuarios de la Situación 2 al 99\% realizaron las tareas casi en su totalidad y para la situación 3 lograron las tareas en un $88 \%$, esto implica que se presenta una interfaz intuitiva y agradable.

Finalmente se aplicó una encuesta de satisfacción "Valoración del Software [13], la cual evalúa siete criterios: Navegación, Interactividad, Inmersión, Usabilidad, Creatividad, Efectividad y Calidad, con una escala de 1 a 5, cuyo promedio obtenido fue de 4.7 (94.37\%), a continuación, se presentan los resultados en la Figura 6.

\section{Conclusiones y trabajo a futuro}

El propósito de este artículo fue presentar una propuesta de una aplicación móvil para poder apoyar a los "Adultos Mayores" en la movilidad de sus actividades cotidianas de manera segura poder compartir su ubicación mediante el uso de un código generado y reconocer el status de los usuarios que han consultado la información, para poder ser localizados de forma más segura, logrando una aplicación intuitiva y agradable. La metodología Programación Extrema y el Diseño Centrado en el Usuario nos permitieron mostrar trabajar de forma más cercana con el usuario y generar algunas versiones previas para que el usuario probara y revisara los avances de acuerdo a sus necesidades y trabajar con un grupo focal de "Adultos Mayores" para centrarnos en una aplicación más agradable y de fácil manejo para este tipo de población. 


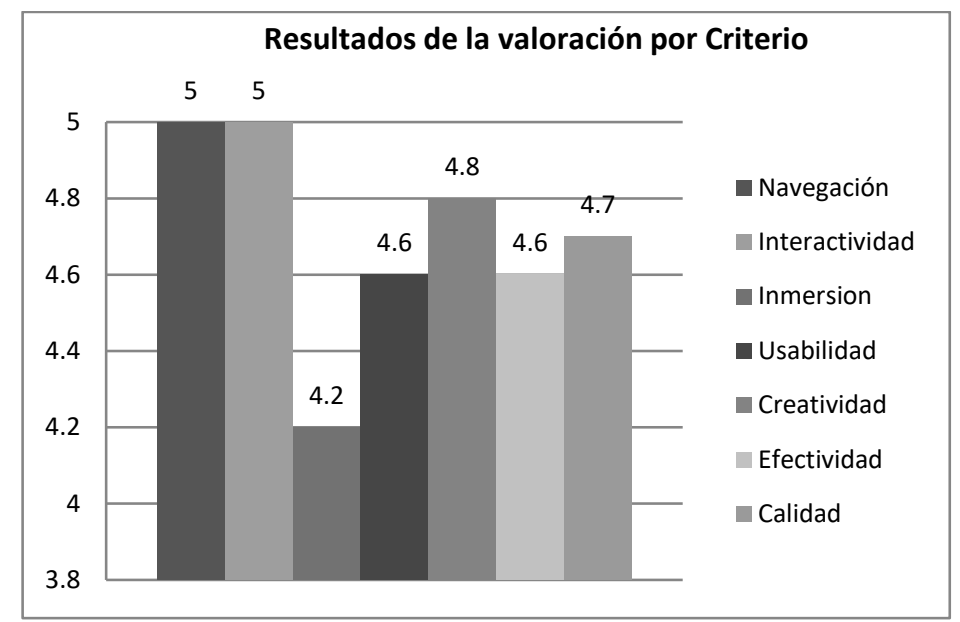

Fig. 6. Resultados de la encuesta de satisfacción.

Una de las aportaciones de esta propuesta de aplicación móvil es la facilidad de exportar la opción del código que contiene las coordenadas para otras aplicaciones y compartir entre los distintos usuarios o familiares de tal manera que los usuarios que utilicen la aplicación puedan ubicar el destino sin necesidad de buscarlo nuevamente, solo bastaría ingresar el código para poder obtener la información y de esta forma llevar un registro de los usuarios en la base de datos, quienes tienen acceso a dicha información por medio de las tecnologías de WebService, PHP, MySql y Java.

Como trabajo futuro se espera incorporar otras pruebas de usabilidad con una muestra mayor de usuarios y una vez que se tenga una versión estable la aplicación, se planea incorporar al "Portal de Discapacitados" a la sección de Aplicaciones Accesibles para uso de la comunidad y apoyo a grupos vulnerables.

\section{Referencias}

1. Muñoz, C.: Bienestar Subjetivo y Actividad Social con Sentido Histórico en Adultos Mayores. Revista Hacia la Promoción de la Salud, http://google.redalyc.org/articulo.oa?id=309131077002, 13-26

2. Naciones, U.: La sostenibilidad y la inclusión de las personas mayores en el entorno urbano. http://www.un.org/es/events/olderpersonsday/ (2015)

3. Alcantud, F.: Estudiantes con Discapacidades Integrados en los Estudios Universitarios. Notas para su Orientación (1995)

4. Naciones, U.: Convención sobre los Derechos de las Personas con Discapacidad. http://www. un.org/esa/socdev/enable/documents/tccconvs.pd f $(2015)$

5. Gil, S.: Cómo hacer Apps Accesibles. Centro de Referencia Estatal de Autonomía Personal y Ayudas Técnica, http://www.ceapat.es/ceapat_01/index.htm, 5-85 (2013)

6. Cuello, J.: Diseñando apps para móviles. http: / /appdesignbook . com/ (2015) 
Prototipo de una aplicación móvil accesible para apoyar a la localización de adultos mayores ...

7. Fuentes, D., Opitz, I., Oro, N.: Análisis de Aplicación Móvil basada en Geolocalización. http://profesores.elo.utfsm.cl/ agv/elo322/1s16/projects/rep orts/Informe_G14_Elo322.pdf (2015)

8. Life360. https://play.google.com/store/apps/details?id=com.life 360. android.safetymapd\&hl=es (2015)

9. Bernardos, A., Besada, J., Casar, C.: Tecnologías de localización, ETS Ingenieros de Telecomunicación. Universidad Politécnica de Madrid, http://www. upm.es/sfs/Rectorado/Organos\%20de\%20Gobierno/Cons ejo\%20Social/Actividades/tecnologias_servicios_para_sociedad informacion.pdf (2005)

10. Hassan-Montero, Y., Ortega-Santamaría, S. Informe APEI sobre Usabilidad. Gijón: Asociación Profesional de Especialistas en Información. http://www. nosolou sabilidad.com/manual/index.htm (2009)

11. Extreme Programming. http://www. extremeprogramming.org/ (2013)

12. Barry, D.: Service Architecture. (s.f.), http://www.servicearchitecture.com/articles/web-services/soap.html

13. Acuña, A., Romo, M.: Diseño Instruccional Multimedia. Pearson Education, México (2011) 